\title{
ANALYSIS OF RESONANT TUNNELING FOR PARABOLIC DOUBLE BARRIER STRUCTURE
}

\author{
E. KACZMAREK \\ Institute of Physics, Polish Academy of Sciences \\ Al. Lotników 32/46, 02-668 Warszawa, Poland
}

In this paper the resonant tunneling through the double barrier structure with the parabolic quantum well is studied theoretically. The transmission coefficient for such a structure as a function of applied voltage and the current-voltage characteristics are calculated and compared with those for the double barrier structure with the rectangular quantum well. The conclusion is that the resonant tunneling through parabolic double barrier structure can be used as a method of determination of the conduction band offset of the barrier and the well materials.

PACS numbers: $73.40 . \mathrm{Gk}$

Recently, quantum structures with graded confining potential have received a great deal of interest, both from the point of view of their physical properties as well as from the point of view of their potential applications. The parabolic quantum-well structures were studied mainly by means of photoluminescence excitation spectroscopy [1]. This method allows us to determinate the partitioning of the energy gap discontinuities between the conduction and the valence bands. The resonant tunneling through the GaAs-AlGaAs double barrier structures (DBS) with a wide parabolic quantum well was also reported $[2,3]$.

Since its first observation in 1974 [4] the resonant tunneling has been studied theoretically only for the double barrier structures with a rectangular well between the barriers. The purpose of the present paper is an analysis of this phenomenon for the double barrier structures with the parabolic well. The considered one-dimensional potential profile is shown in Fig. 1 for unbiased and biased structures. The curvature of the well is determined by the height $V_{0}$ of the barrier and the half-width $w$ of the well.

The global transmission coefficient, $T_{\mathrm{G}}$, for the structure under constant electric field, $F$, was calculated by the standard method, i.e., by matching the electron wave functions and their derivatives at the points of discontinuity of potential [5]. The electron wave functions were taken as linear combinations of the Airy functions in the barrier regions

$$
\psi_{\mathrm{b}}(x)=A_{\mathrm{b}} \operatorname{Ai}\left(z_{0}-z\right)+B_{\mathrm{b}} \operatorname{Bi}\left(z_{0}-z\right)
$$

where

$$
z=\left(\frac{2 m_{\mathrm{e}} e F}{\hbar^{2}}\right)^{1 / 3} x \quad \text { and } \quad z_{0}=\left(\frac{2 m_{\mathrm{e}} e F}{\hbar^{2}}\right)^{1 / 3} \frac{V_{0}-E}{e F}
$$




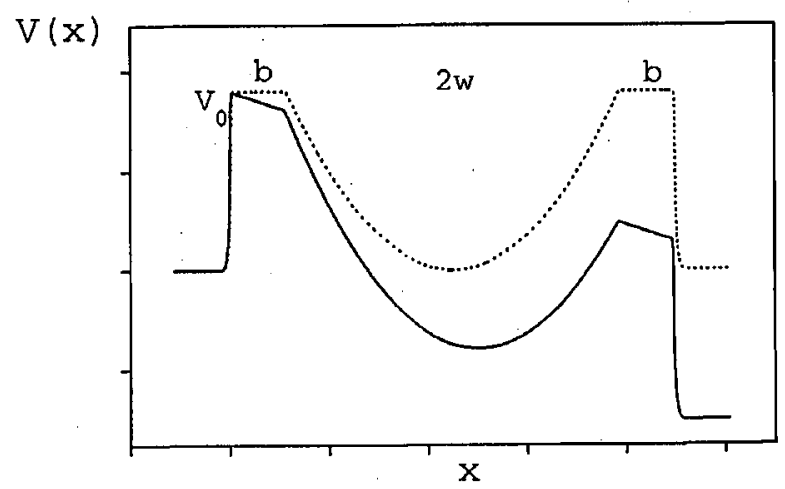

Fig. 1. Potential-energy profiles of the parabolic DBS without (dotted line) and with (solid line) an applied bias.

and of the confluent hypergeometric functions in the parabolic well $[6,7]$ :

$$
\psi_{\mathrm{w}}(x)=\left[A_{\mathrm{w}} M\left(a, \frac{1}{2}, \eta\right)+B_{\mathrm{w}} \eta^{1 / 2} M\left(\frac{1}{2}+a ; \frac{3}{2}, \eta\right)\right] \exp \left(-\frac{\eta}{2}\right)
$$

where

$$
\begin{aligned}
& \eta=\frac{1}{w} \sqrt{\frac{2 m_{\mathrm{e}} V_{0}}{\hbar^{2}}}(x-b-w)^{2}, \\
& a=\frac{1}{4}\left(1-\sqrt{\frac{2 m_{\mathrm{e}} w^{2}}{\hbar^{2}}} \frac{E+e F(b+w)+(e F w)^{2} / 4 V_{0}}{\sqrt{V_{0}}}\right) .
\end{aligned}
$$

Here $m_{e}$ and $E$ denote, respectively, the effective mass and the energy of tunneling electron.

The expression for the transmission coefficient, $T_{\mathrm{G}}$, is a more complicated function of tunneling electron energy, structure parameters and an applied electric field than that for DBS with the rectangular well. However, the characteristic features of the transmission coefficient are the same as for DBS with the rectangular well. The example is shown in Fig. 2 where $\ln T_{\mathrm{G}}$ is plotted as a function of tunneling electron energy for unpolarized GaAs-AlGaAs structure. The sharp maxima of transmission coefficient correspond to resonant tunneling through the equidistant energy levels in the parabolic well. In a wide range of the barrier heights and parabolic well widths the energy distance between adjacent maxima is equal to that calculated in the infinite barrier approximation

$$
\Delta E=\hbar \omega=\hbar \sqrt{2 V_{0} / w^{2} m_{e}} \text {. }
$$

To illustrate how the oscillation character of the global transmission coefficient influences the current-voltage characteristics of the considered structure we have calculated the tunneling current for several sets of the structure parameters using the well-known expression [8]

$$
j(V)=\frac{e}{2 \pi^{2}} \frac{m^{*}}{\hbar^{3}} k_{\mathrm{B}} T \int_{0}^{\infty} T_{\mathrm{G}}(E, V) \ln \frac{1+\exp \left[\left(E_{\mathrm{F}}-E\right) / k_{\mathrm{B}} T\right]}{1+\exp \left[\left(E_{\mathrm{F}}-E-V\right) / k_{\mathrm{B}} T\right]} \mathrm{d} E .
$$




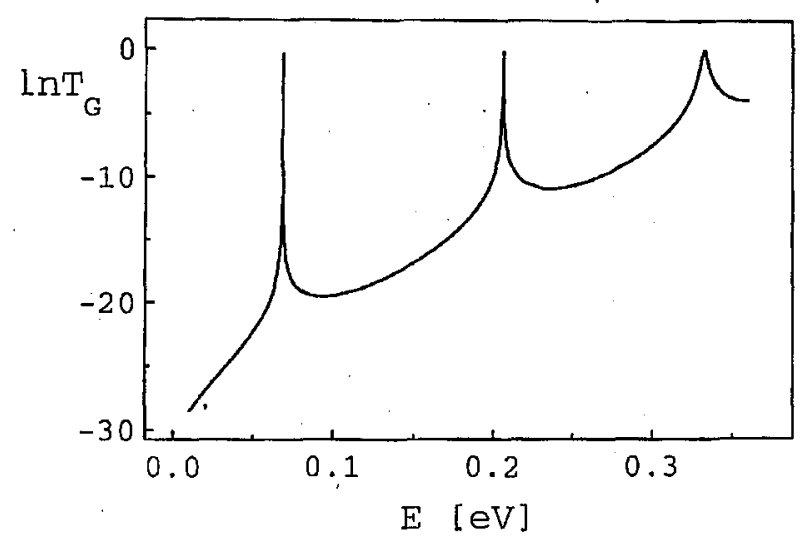

Fig. 2. Transmission coefficient $T_{G}$ as a function of tunneling electron energy $E$ $\left(V_{0}=0.36 \mathrm{eV}, b=56 \AA, w=56 \AA\right)$.

Here, $V$ denotes the potential drop over the whole structure and $E_{\mathrm{F}}$ is the Fermi energy in the emitter.

Generally, the tunneling current has a resonant character, i.e., it increases suddenly whenever a successive level in the parabolic well, pulled down by applied electric field, crosses the Fermi level $E_{\mathrm{F}}$ in the emitter, and next decreases when the level passes by the bottom of the emitter, conduction band. However, the pronounced current peak with a large peak-to-valley ratio can be observed only for the parabolic structures with very thin barriers and a relatively narrow well. The reason is that the value of the resonant current in maximum depends on the barrier width, and the effective barrier width for parabolic DBS is, especially for the first two levels in the quantum well, considerably larger than that at the top of the barrier. The example of calculated $j(V)$ characteristic for the $\mathrm{GaAs}^{-} \mathrm{Al}_{0.4} \mathrm{Ga}_{0.6} \mathrm{As}$ structure with a barrier consisting of $10 \mathrm{ML}$ and the parabolic well consisting of $40 \mathrm{ML}$ is plotted in Fig. 3a. The characteristic for the $\mathrm{Al}_{0.4} \mathrm{Ga}_{0.6} \mathrm{As}$ DBS with the rectangular well ( $b=w=20 \mathrm{ML}$ ) is also shown for comparison. The peak-to-valley ratios for both characteristics are comparable, although the barrier widths differ by the factor of two.

The $j(V)$ characteristics for DBS with wide parabolic wells display several equally spaced maxima for the voltages equal to $V_{1}, V_{2}$, and so on. The number of peaks depends mainly on the width of the parabolic well. The difference $e\left(V_{i+1}-V_{i}\right)$ is, in good approximation, equal to $2 \Delta E$, where $\Delta E$ is given by Eq. (3). For high voltages one may expect departures from this simple rule because of a large deformation of the structure by high electric field. The $\Delta E$ depends on the conduction band offset $V_{0}$ between the barrier and the well materials. Thus, this significant structure parameter can be easily determined from the current-voltage characteristic for the parabolic DBS. An example of the calculated $j(V)$ characteristic with two resonant maxima is shown in Fig. $3 \mathrm{~b}$. In this case the parabolic well consists of $60 \mathrm{ML}$, the other structure parameters are as in Fig. 3a. The energy levels in the well are separated by $107 \mathrm{meV}$, whereas the calculated difference of potential drops at the current maxima is equal to $210 \mathrm{mV}$. 

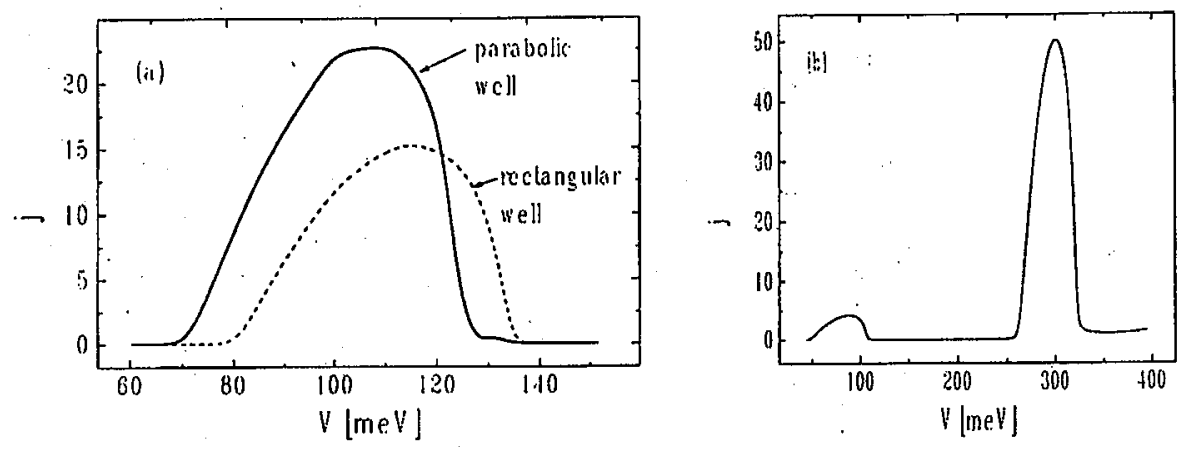

Fig. 3. Calculated $j(V)$ characteristics: (a) for the thin parabolic (solid line) and the rectangular (dashed line) double barrier structures. The barrier heights and the well widths are the same for both structures, the barrier width for the parabolic DBS is equal to half of the barrier width for the rectangular DBS; (b) for the wider parabolic DBS. The distance between the two current maxima is equal to $0.21 \mathrm{~V}$, the energy difference between adjacent levels in the well is $0.107 \mathrm{eV}$.

The results of our simple model calculations agree well with the experimental data for the AlAs-GaAs parabolic double barrier structures [2, 3].

- Summarizing, the resonant tunneling through parabolic double barrier structures can be used as a method of determination of conduction band offset. It would be useful for novel quantum well structures like CdMgTe-CdTe [9], for which the band parameters of barrier material are not well known. In order to observe several sharply outlined resonant transitions the barriers in DBS should be as thin as possible $(\approx 10 \mathrm{ML})$ and the well should be relatively wide $(80-100 \mathrm{ML})$.

\section{References}

[1] T. Wojtowicz, M. Kutrowski, M. Surmạ, K. Kopalko, G. Karczewski, J. Kossut, M. Godlewski, P. Kossacki, Nguyen The Khoi, Appl. Phys. Lett. 68, 3326 (1996).

[2] S. Sen, F. Capasso, A.C. Gossard, R.A. Spah, A.L. Hutchinson, S.N.G. Chu, Appl. Phys. Lett. 51, 1428 (1987).

[3] S.Y. Chou, J.S. Harris, Jr., Appl. Phys. Lett. 52, 1422 (1988).

[4] L.L. Chang, L. Esaki, R. Tsu, Appl. Phys. Lett. 24, 593 (1974).

[5] E.O. Kane, in: Tunneling Phenomena in Solids, Eds. E. Burstein, D. Lundquist, Plenum Press, New York. 1969, p. 1.

[6] W.-P. Yuen, Phys. Rev. B 48, 17316 (1993).

[7] Handbook of Mathematical Functions, Eds. M. Abramowitz, I.A. Stegun, Dover Publications Inc., New York.

[8] R. Tsu, L. Esaki, Appl. Phys. Lett. 22, 562 (1973).

[9] T. Wojtowicz, M. Kutrowski, G. Karczewski, J. Kossut, Acta Phys. Pol.' A 94, 199 (1998). 